\title{
Influência do Amendoim Forrageiro (Arachis pintoi) e do Feijão-de-Porco (Canavalia ensiformis) como plantas de cobertura no controle de erosão hídrica sob chuva simulada
}

\author{
Larissa Adrielly Rodrigues de Sousa ${ }^{1 *}$, Alain Gimenez Molnar ${ }^{1}$, Danilo Ribeiro da Costa ${ }^{2}$
}

\begin{abstract}
RESUMO: A erosão é responsável pela degradação do solo através do arraste das partículas para um local diferente de sua origem. O experimento foi realizado em um Cambissolo, localizado no Núcleo de Estudos Ambientais de Sorocaba (NEAS), composto por 9 parcelas (1,10 m x 2,65 m), definido por três espaçamentos para as espécies, sendo: 15 x 15 cm, $25 \times 25 \mathrm{~cm} \mathrm{e} 40 \times 40 \mathrm{~cm}$ e três testemunhas com solo desnudo. Para quantificação das perdas de solo e avaliação das espécies, utilizaram-se coleta das folhas para análise foliar, quantificação da perda de água e solo, medição da altura das plantas e observação do índice de cobertura (IC) semanalmente. Levando em consideração todas as medições realizadas, o feijão-de-porco de espaçamento 15 x $15 \mathrm{~cm}$ obteve melhores resultados para proteção do solo em curto prazo devido ao seu crescimento inicial rápido, e o amendoim 15 x $15 \mathrm{~cm}$ apresentou melhores medições de proteção do solo em longo prazo devido ao crescimento inicial lento e à sua capacidade de rebrota.
\end{abstract}

Palavras-chave: conservação do solo, perda de solo, adubos verdes

\section{Influence of Peanut (Arachis pintoi) and Jackbean (Canavalia ensiformis) as cover plants in the control of water erosion under simulated rain.}

\begin{abstract}
The erosion is responsible for soil degradation, starting with the detachment, and subsequently transport and drag the soil particles to a location that is different from its origin. The experiment was conducted in a Cambisol,

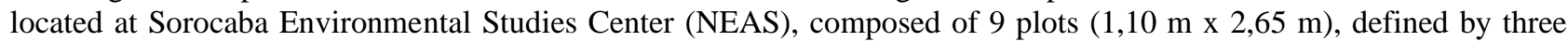
spacings for the species being: $15 \times 15 \mathrm{~cm}, 25 \times 25 \mathrm{~cm}, 40 \times 40 \mathrm{~cm}$ and three witnesses with bare soil. For the quantification of soil losses and to avaluate the behavior of the species, leaf collection was used for leaf analysis, quantification of water and soil loss, measuring the height of plants and observation of the index of coverage (IC) weekly. Taking into consideration all measurements, JackBean with 15 x $15 \mathrm{~cm}$ spacing was considered the best option for soil protection in the short term due to its initial growth and the peanut, although it has a slower initial growth, even in $15 \times 15 \mathrm{~cm}$ spacing presented good measurements, in addition to being able to be used for soil protection in the long term of time, due to its capacity of regrowth.

Keywords: soil conservation, soil loss, green fertilizers
\end{abstract}

\section{INTRODUÇÃO}

Durante o desenvolvimento da agricultura no mundo as formas de manejo desenvolveram-se em diferentes aspectos tendo como foco melhorar a qualidade de produção das culturas implantadas, esse avanço intensificou o uso da terra que promoveu ao longo do tempo um aumento da degradação dos solos (ESPINOLA; GUERRA; ALMEIDA, 1997).

Segundo Bertoni e Lombardi Neto (2012), a perda da disponibilidade, que acarreta na perda de produtividade, é influenciada pela exposição do solo, ou seja, dentro de um processo produtivo o solo encontra-se sem cobertura vegetal. A erosão hídrica é considerada o tipo de erosão que mais causa desgastes físicos aos solos (CASTRO et al., 2011), pois seu início se dá com o impacto da gota de chuva no solo exposto, quebrando os agregados em partículas menores e posteriormente à condução dessas partículas para um local diferente de sua origem (BERTONI E NETO, 2012).

\footnotetext{
${ }^{1}$ Universidade de Sorocaba

${ }^{2}$ Universidad Federal de São Carlos

E-mail: lari-adrielly@hotmail.com
} 


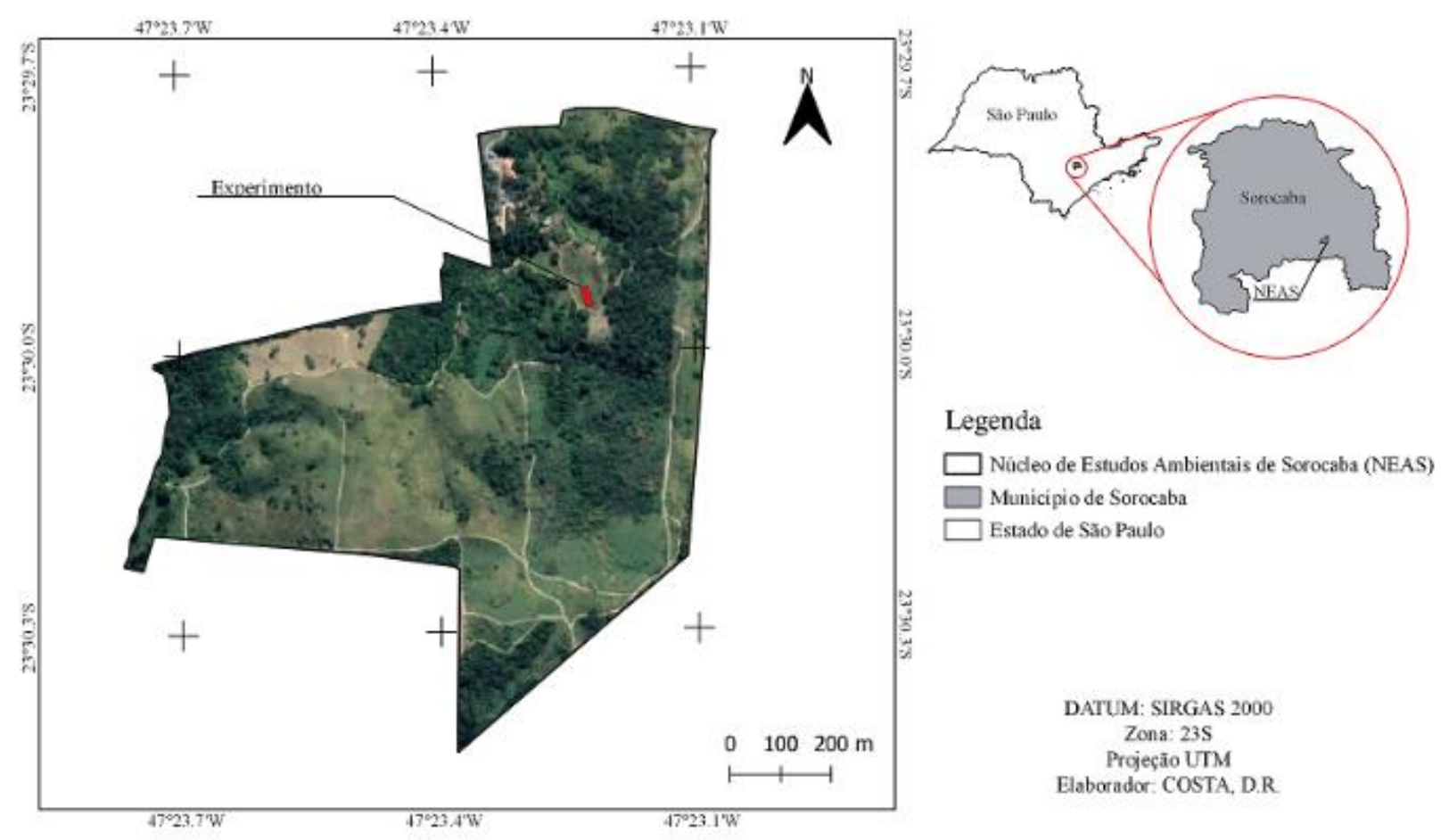

Figura 1 - Localização do Núcleo de Estudos Ambientais (NEAS), Sorocaba - SP.

A utilização de práticas conservacionistas favorece o controle da erosão hídrica devido à produção de biomassa vegetal, reduzindo o escoamento da água e a perda de solo (PENTEADO, 2010). Dentre as técnicas de conservação às caráter vegetativo demonstram importância por promove a proteção do solo do impacto da chuva e fornece ao solo nutrientes e biomassa a partir do uso de plantas leguminosas (CARVALHO et al., 2013; MATHEIS et al., 2006)

O objetivo deste trabalho baseia-se observar o uso do Amendoim Forrageiro (Arachis pintoi) e do Feijão-de-porco (Canavalia ensiformis) no controle da erosão hídrica, avaliar o desenvolvimento das espécies e a influência sobre a perda de solo.

\section{MATERIAL E MÉTODOS}

O experimento foi realizado no município de Sorocaba, no Núcleo de Estudos Ambientais de Sorocaba (NEAS), figura 1. Segundo Peçanha et al. (2016), a UNISO campus Cidade Universitária apresenta uma área de cerca de 135,52 ha (56 alqueires), composta predominantemente por Cambissolo e apresenta variação de altitude de 615 a 689 metros. Segundo classificação de KOPPEN o clima é do tipo Cwa tropical úmido onde apresenta verões quentes e invernos secos.

Para a avaliação das perdas de solo, foram montadas parcelas com dimensões de $2,91 \mathrm{~m}^{2}$ $(1,10 \mathrm{~m} \times 2,65 \mathrm{~m})$. Foram utilizadas chapas galvanizadas para direcionar o fluxo da água em direção ao ponto coletor, devido à declividade da área, sendo a média de $15 \%$.

$\mathrm{Na}$ extremidade inferior de cada parcela foi instalado um tubo de PVC de 4" para coletar a água e o solo, estes serão direcionados ao coletor final de 15 litros (figura 2).

A semeadura do feijão-de-porco (FP) foi realizada através de sementes em covas de $5 \mathrm{~cm}$ de profundidade, e o amendoim-forrageiro $(\mathrm{AF})$ através de mudas transplantadas equivalente ao tamanho do feijão-de-porco, média de aproximadamente $6 \mathrm{~cm}$ nos três diferentes espaçamentos, sem utilização de adubação.

A avaliação de perda de solo e de água nas parcelas, foram realizadas sob chuva simulada semanalmente através de um regador de 10 litros com crivo. Para a quantificação das perdas de água (Psa) e solo (Pso), cada parcela recebeu $20 \mathrm{~mm}$ de água, ou seja, 20 litros $/ \mathrm{m}^{2}$.

Ao final da simulação, foram retirados os coletores finais contendo a perda de solo e água pelo processo de escoamento superficial para quantificação. 


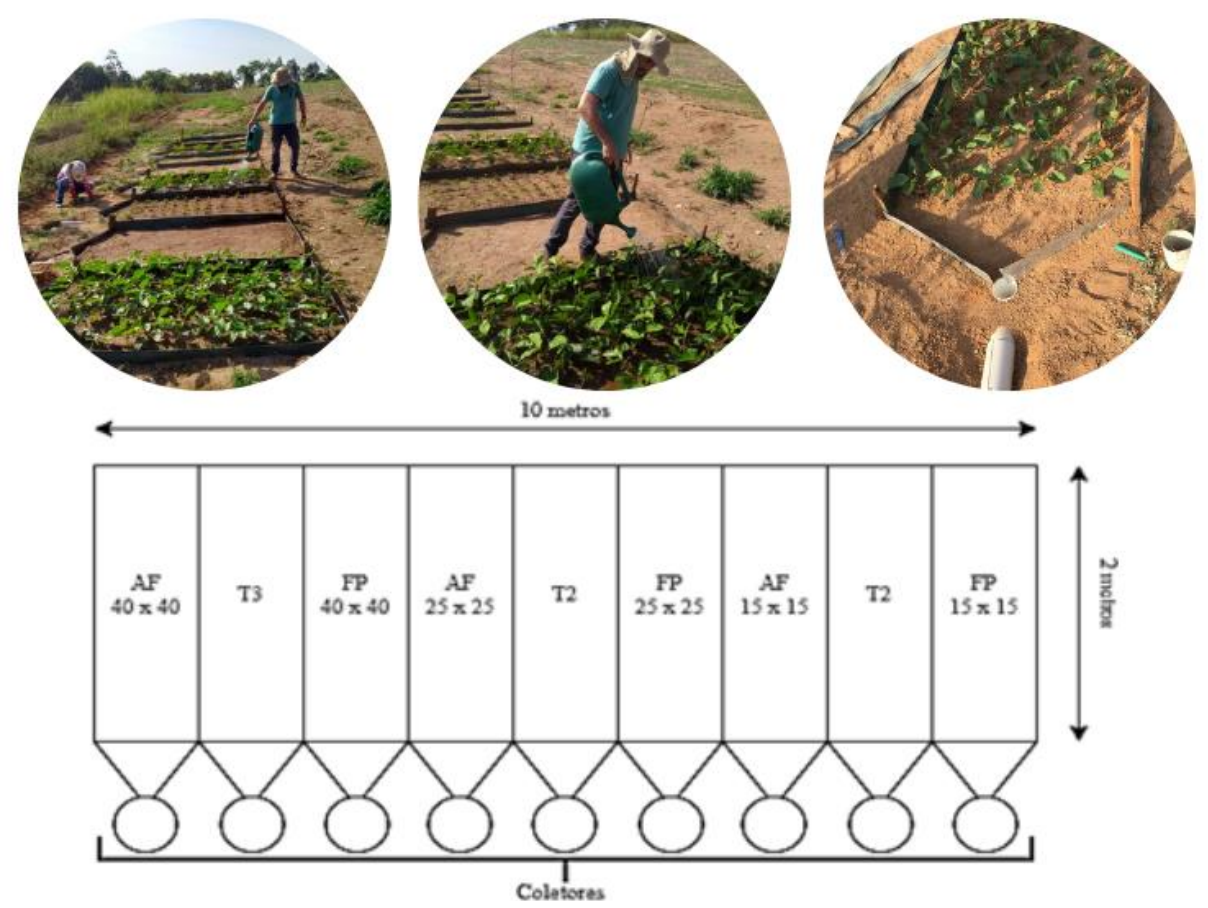

Figura 2 - Parcelas experimentais submetidas à simulação de chuva e coleta de solo e água

Para a determinação do Índice de Cobertura (IC), foi empregada a metodologia definida por (Stocking, 1988), através de um instrumento com orifícios de $8 \mathrm{~mm}$ de diâmetro cada, com espaçamento de $16,66 \mathrm{~cm}$ entre eles, totalizando 12 orifícios. Para esta medição de crescimento das plantas foram mensuradas as alturas e a áreas foliar específica (SLA), através do programa ImageJ®.

\section{Análise de dados}

A análise de dados foi realizada a partir de estatísticas descritivas em relação as variáveis levantadas. E a correlação de Pearson entre as variáveis (alta, $r>0,80$; média, $0,50 \leq \mathrm{r} \leq 0,80$; e baixa, $r<0,50)$.

\section{RESULTADOS E DISCUSSÕES}

Com relação à medições das plantas, observou-se o crescimento expressivo do feijãode-porco comparado ao amendoim forrageiro, devido ao seu crescimento inicial rápido. A variável foliar específica (SLA) foi observada para averiguar a interferência no processo de redução do impacto das gotas de chuva no solo.

Os maiores resultados foram do feijão de porco, conforme figura 3 . (a)

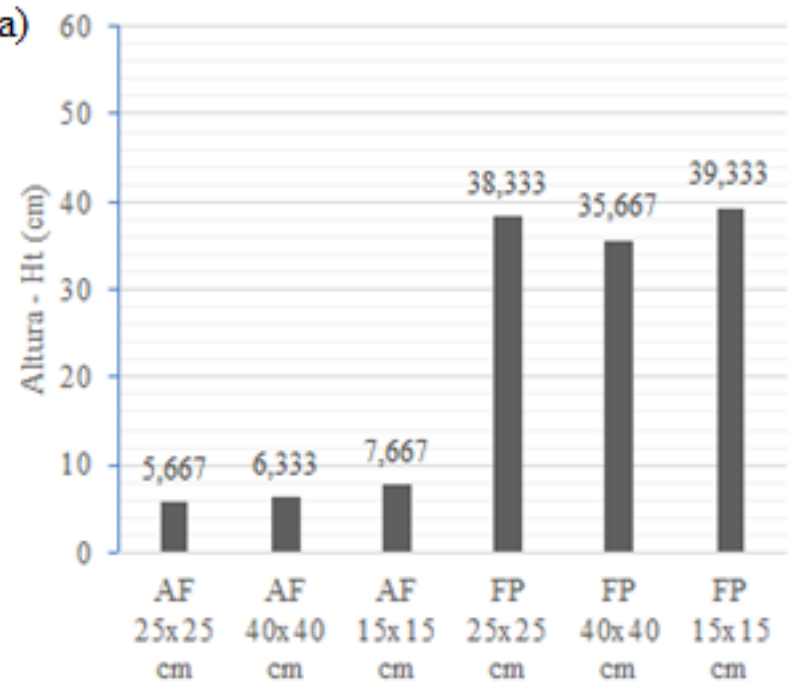

(b)

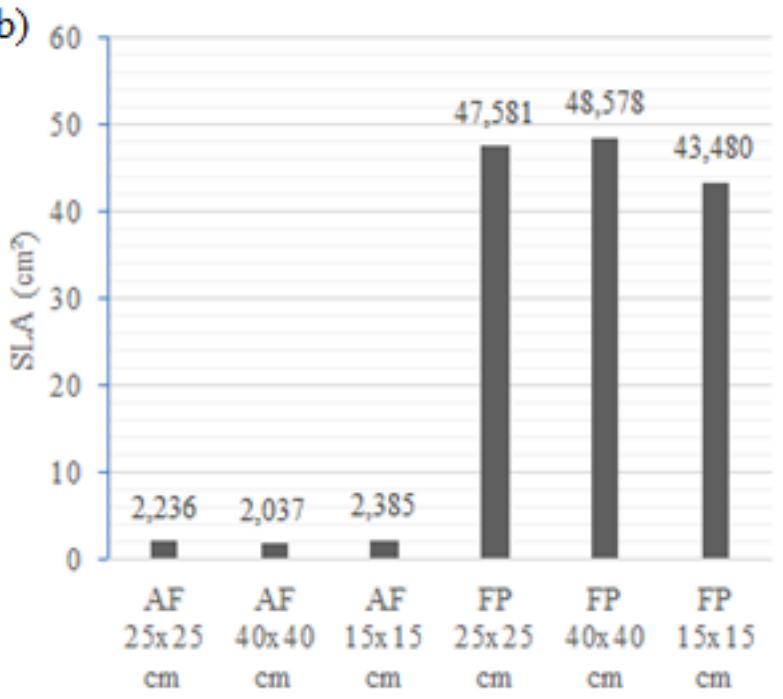

Figura 3 - (a) Crescimento em Altura (Ht) em centímetros; (b) Área foliar (SLA) das espécies em relação aos tratamentos. 
Para determinação do Índice de Cobertura (IC), Lopes et al (1987), estipula que plantas que possuem IC maior que $30 \%$ é capaz de reduzir as perdas de solo por erosão hídrica. $\mathrm{O}$ feijão-de-porco, desde o início obteve bons resultados nos espaçamentos $15 \times 15 \mathrm{~cm}$ e $25 \times$
$25 \mathrm{~cm}$. Já o amendoim forrageiro, teve o melhor índice de cobertura no espaçamento $15 \times 15 \mathrm{~cm}$, conforme mostra a tabela 1.e 25 x $25 \mathrm{~cm}$. Já o amendoim forrageiro, teve o melhor índice de cobertura no espaçamento $15 \times 15 \mathrm{~cm}$, conforme mostra a tabela 1 .

Tabela 1 - Relação do Índice de Cobertura (IC) em relação ao tratamento ao longo do tempo das medições

\begin{tabular}{ccccc}
\hline Parcela & $\mathbf{l}^{\mathbf{2}}$ M ediçño & $\mathbf{2}^{2}$ Mediç̃o & 3 $^{\mathbf{2}}$ Mediç̃o & Média \\
\hline FP 15 x 15 cm & $81,25 \%$ & $95,83 \%$ & $96,87 \%$ & $91 \%$ \\
FP 25 X 25 cm & $67,70 \%$ & $78,12 \%$ & $82,29 \%$ & $76 \%$ \\
FP 40 X 40 cm & $36,45 \%$ & $56,25 \%$ & $52,08 \%$ & $48 \%$ \\
AF 15 X 15 cm & $43,75 \%$ & $60,41 \%$ & $67,70 \%$ & $57 \%$ \\
AF 25 X 25 cm & $19,79 \%$ & $37,50 \%$ & $50,00 \%$ & $36 \%$ \\
AF 40 X 40 cm & $09,13 \%$ & $11,45 \%$ & $15,62 \%$ & $12 \%$ \\
\hline
\end{tabular}

Foi possível observar o comportamento das variáveis entre si pela análise de correlação de Pearson, figura 4.

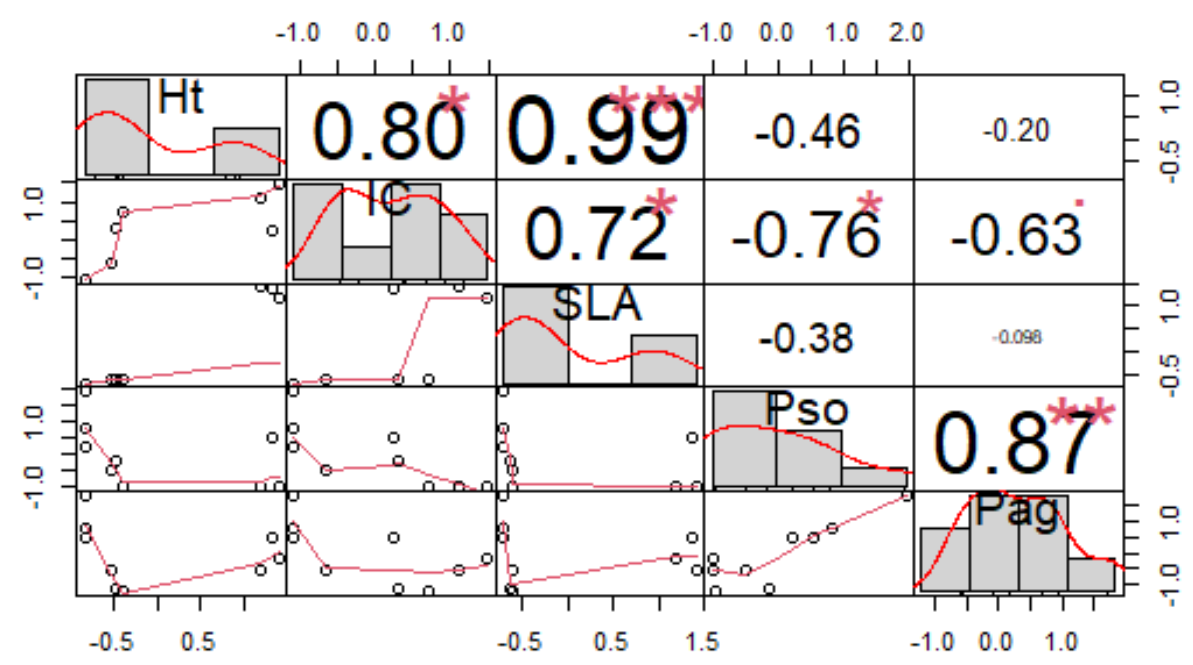

Figura 4 - Análise de Correlação entre as variáveis: Ht: Altura total; IC: Índice de Cobertura; SLA: Área Foliar; Pso: Perda de solo em gramas; Psa: Perda de água em litros.

A análise de correlação demonstrou que o Índice de Cobertura (IC) relaciona-se negativamente com a Perda de Solo (Pso, -0,76) e Perda de Água (Psa, -0,63), demonstrando menores valores de cobertura vegetal aumenta a perda do solo e da água. Lopes et al. (1987), estipula que plantas que possuem IC maior que $30 \%$ são capazes de reduzir as perdas de solo por erosão hídrica. Por fim foi possível mensurar a perda de solo em a cada uma das parcelas (figura 5). 


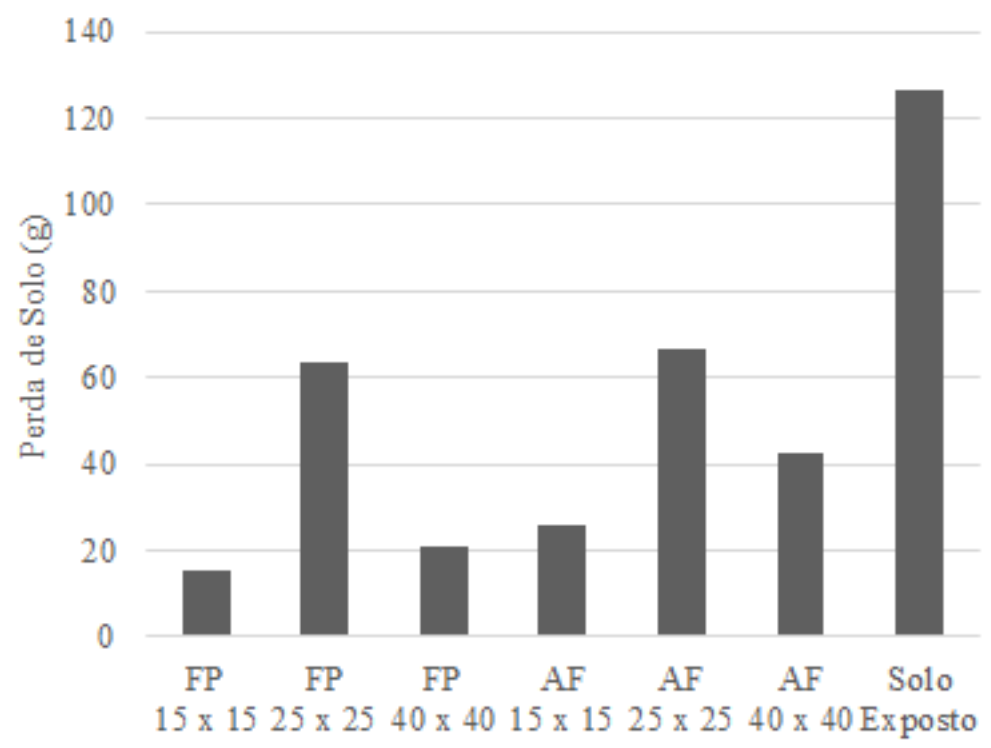

Figura 5 - Perda média de solo em gramas (g) nas parcelas experimentais.

A maior perda de solo está nas parcelas que foram deixadas com solo exposto sendo um resultado esperando uma vez que a falta de cobertura vegetal afeta diretamente $o$ desprendimento das partículas (BERTONI E NETO, 2012; GUERRA, 2012).

Para ambas as espécies a sua implantação em menor espaçamento promoveu menor perda de solo sendo notório que independente do espaçamento e da espécie o recobrimento do solo diminuiu o impacto direto das gostas de chuva. Dentre as espécies a que melhor respondeu a proteção do solo foi o Feijão-deporco (FP) dentre os motivos que possa responder seu melhor desempenho foi a taxa de crescimento e cobertura do solo maior em comparação com Amendoim-Forrageiro (AF). Missio; Debiasi; Martins (2004) em estudos de crescimento de plantas de adubação verde demonstraram que o Feijão-de-porco apresenta um melhor desempenho de recobrimento do solo ao decorrer do seu desenvolvimento.

\section{CONCLUSÕES}

Tendo em vista os parâmetros analisados, conclui-se que o Feijão-de-Porco com espaçamentos $15 \times 15 \mathrm{~cm}$, obteve melhores resultados devido ao seu crescimento inicial ser mais rápido.

Já o Amendoim forrageiro, foi observado que a planta com espaçamento 15 x $15 \mathrm{~cm}$ apresentou melhores resultados. Além disso, a presença das duas culturas com bom índice de cobertura, sendo o feijão-de- porco a curto prazo e o amendoim forrageiro a longo prazo gerou uma menor perdas de solo e água devido à capacidade de sua área foliar impedir o impacto das gotas diretamente no solo, agindo assim como barreiras.

\section{AGRADECIMENTOS}

A Universidade de Sorocaba (UNISO) por disponibilizar o local para instalação e análises do experimento, a Universidade Federal de São Carlos, campus Sorocaba, por disponibilizar as sementes de adubação verde utilizadas.

\section{REFERENÇAS BIBLIOGRÁFICAS}

BERTONI, J.; NETO, F. L. CONSERVAÇÃO DO SOLO. 8. Ed. São Paulo: Ícone, 2012. 355 p.

CARVALHO, W.P.; CARVALHO, G.J.; NETO, D. O. A.; TEIXEIRA, L. G. V. Desempenho agronômico de plantas de cobertura usadas na proteção do solo no período de pousio. Pesq. agropec. bras., Brasília, v.48, n.2, p.157-166, 2013.

ESPÍNDOLA, J.A.A.; GUERRA, J.G.M.; ALMEIDA, D.L. de. Adubação verde: Estratégia para uma agricultura sustentável. Seropédica, Embrapa-Agrobiologia, 1997. $20 \mathrm{p}$.

LOPES, P.R.; COGO, N.P.; LEVIEN, R. Eficácia relativa de tipo e quantidade de resíduos culturais espalhados uniformemente sobre o solo na redução da erosão hídrica. Revista Brasileira de Ciência do Solo, Viçosa, v.11. n.1, p.71-75, jan./abril. 1987. 
MATHEIS, H.A.S.M.; AZEVEDO, F.A. de; VICTÓRIA FILHO, R. Adubação verde no manejo de plantas daninhas na cultura de citros. Laranja, v.27, p.101-110, 2006.

MISSIO, E. L.; DEBIASI, H.; MARTINS, J. D. Comportamento de leguminosas para cobertura do solo, adubação verde e controle de plantas daninhas. Pesq. Agrop. Gaúcha. Porto Alegre, v.10, n.1-2, p. 129-136, 2004.

PEÇANHA, M. P.; FREITAS, N. P.; DIAS, A.; GARCIA, J. P. M.; DIGIAMPIETRI, E. A.; DIGIAMPIETRI FILHO, E. A.; COSTA, M. DE A.; REAL, M. G. C.; GOBBO, P. R. S.; SOUZA, N. R.; TSERMAZIDES, K. G.; ANDRADE JÚNIOR, I. P.; ASSIS, F. S. Caracterização ambiental do Câmpus Raposo da Universidade de Sorocaba - Uniso. Revista de Estudos Universitários - REU, v. 26, n. 2, 11 nov. 2016.

PENTEADO, Silvio Roberto. Adubos verdes e produção de biomassa: Melhoria e recuperação dos solos. 2. ed. Campinas. Via Orgânica - Fraga Penteado \& Cia Ltda, 2010. 168p.

GUERRA, A.J.T. Processos erosivos no domínio do Cerrado. In: GUERRA, A. J. T., SILVA, A. S. \& BOTELHO, R. G. M (org.). Erosão e Conservação dos Solos: conceitos, temas e aplicações. Bertrand Brasil, Rio de Janeiro, 2012. 340p.

STOCKING, M. A. Assessing vegetative cover and management effect. In: LAL, R. Soil erosion research methods. Iowa: Soil and Water Conservation Society, p.163-167, 1988. 